\title{
Completeness Results for Linear Logic on Petri Nets
}

\author{
Uffe Engberg Glynn Winskel \\ Computer Science Department* \\ Aarhus University \\ Ny Munkegade \\ DK-8000 Aarhus C, Denmark
}

\begin{abstract}
Completeness is shown for several versions of Girard's linear logic with respect to Petri nets as the class of models. The strongest logic considered is intuitionistic linear logic, with $\otimes, \multimap, \&, \oplus$ and the exponential ! ("of course"), and forms of quantification. This logic is shown sound and complete with respect to atomic nets (these include nets in which every transition leads to a nonempty multiset of places). The logic is remarkably expressive, enabling descriptions of the kinds of properties one might wish to show of nets; in particular, negative properties, asserting the impossibility of an assertion, can also be expressed.
\end{abstract}

\section{Introduction}

In [EW90] it was shown how Petri nets can naturally be made into models of Girard's linear logic in such a way that many properties one might wish to state of nets become expressible in linear logic. We refer the reader to the [EW90] for more background and a discussion of other work. That paper left open the important question of completeness for the logic with respect to nets as a model. The question is settled here.

\footnotetext{
*e-mail address: \{engberg,gwinskel\}@daimi.aau.dk, fax: ++45 86135725
} 
We restrict attention to Girard's intuitionistic linear logic. Our strongest result is for the full logic described in [GL87, Laf88], viz. it includes

$$
\otimes, \multimap, \oplus, \& \text {, and ! }
$$

though at a cost, to the purity of the linear logic, of adding quantification over markings and axioms special to the net semantics. For this strongest completeness result, a slight restriction is also made to the Petri nets considered as models; they should be atomic (see definition 22), but fortunately this restriction is one generally met, and even often enforced, in working with Petri nets. The step of considering only atomic nets as models has two important pay-offs: one is that the exponential ! $A$ becomes definable as $A \& \mathbf{1}$, where $\mathbf{1}$ is the unit of $\otimes$; the other is that we can say internally, within the logic, that an assertion is not satisfied - the possibility of asserting such negative properties boosts the logic's expressive power considerably. We can achieve completeness for more modest fragments of the logic without extra axioms and with respect to the entire class of nets as models (see section 6).

The work here contrasts with other approaches to linear logic on Petri nets in that they either apply only to much smaller fragments of the logic such as the $\otimes$-fragment (cf. [GG89]), or use the transitions of a Petri net to freely generate a linear-logic theory (cf. [MOM91]), in which case the logic becomes rather inexpressive, and in particular cannot capture negative properties, or they don't address completeness at all.

While it is claimed that this paper together with [EW90] help in the understanding of linear logic, it is also hoped that, through these results, linear logic will come to be of use in reasoning about Petri nets and, through them, in concurrent computation.

\section{Linear Intuitionistic Logic}

The connectives of linear intuitionistic logic are:

$\otimes$ tensor, with unit 1, called one,

\& conjunction, with unit $\mathrm{T}$, called true,

$\oplus$ disjunction, with unit $F$, called false. 
We take as the definition of linear intuitionistic logic the proof rules presented in [GL87, Laf88]:

\begin{tabular}{|c|c|c|c|c|}
\hline \multicolumn{5}{|l|}{ Structural rules } \\
\hline$\overline{A \vdash A}$ (identity) & \multicolumn{2}{|c|}{$\frac{\Gamma \vdash A \Delta, A \vdash B}{\Gamma, \Delta \vdash B}(\mathrm{cut})$} & $\frac{\Gamma, A, B, \Delta \vdash C}{\Gamma, B, A, \Delta \vdash C}(\mathrm{e}$ & xchange) \\
\hline ogical rules & & & & \\
\hline$\Gamma \vdash A \quad \Delta \vdash B$ & $\Gamma, A, B \vdash C$ & $\Gamma \vdash$ & & \\
\hline$\overline{\Gamma, \Delta \vdash A \otimes B}^{(\vdash \otimes)}$ & $\overline{\Gamma, A \otimes B \vdash C}(\otimes \vdash)$ & $\Gamma, 1$ & $-A$ & $\overline{\vdash 1}$ \\
\hline$\Gamma \vdash A \quad \Gamma \vdash B$ & $\Gamma, A \vdash C$ & & $B \vdash C$ & \\
\hline$\Gamma \vdash A \& B$ & $\overline{\Gamma, A \& B \vdash C}$ & $\overline{\Gamma, A}$ & $\& B \vdash C$ & $\overline{\Gamma \vdash \mathrm{T}}$ \\
\hline$\Gamma \vdash A$ & $\Gamma \vdash B$ & $\Gamma, A$ & $-C \Gamma, B \vdash C$ & \\
\hline$\overline{\Gamma \vdash A \oplus B}^{(\vdash \oplus \ell)}$ & $\overline{\Gamma \vdash A \oplus B}(\vdash \oplus r)$ & & $4 \oplus B \vdash C$ & $\overline{\Gamma, \mathrm{F} \vdash A}$ \\
\hline$\Gamma, A \vdash B$ & $\underline{\Gamma \vdash A \quad \Delta, B \vdash C}$ & & & \\
\hline$\overline{\Gamma \vdash A \multimap B}$ & $\overline{\Gamma, \Delta \cdot A \multimap B \vdash C}$ & & & \\
\hline
\end{tabular}

We use $\Gamma$ as an abbreviation for a (possibly empty) sequence $A_{1}, \ldots, A_{n}$ of assumption formulae.

The absence of the rules for thinning and contraction is compensated, to some extent, by the addition of the logical operator "of course". In [GL87, Laf88] this operator is presented with the following proof rules (stronger than those in [Gir87]):

"Of course" rules

$$
\begin{gathered}
\overline{! A \vdash A} \quad \overline{! A \vdash \mathbf{1}} \quad \overline{! A \vdash ! A \otimes ! A} \\
\frac{B \vdash A}{B \vdash \mathbf{1} \quad B \vdash B \otimes B} \\
B \vdash ! A
\end{gathered}
$$

Given a proposition $A$, the assertion of $! A$ has the possibility of being instantiated by the proposition $A$, the unit 1 or $! A \otimes ! A$, and thus of arbitrarily many assertions of $! A$. 


\section{Quantale Interpretation}

As recognised by several people [AV88, Yet90, Ros90, Sam], quantales ${ }^{1}$ provide an algebraic semantics for linear intuitionistic logic. Quantales are to linear intuitionistic logic as complete Heyting algebras are to intuitionistic logic. A quantale is a commutative monoid on a complete join semilattice. Spelled out:

Definition 1 A quantale $\mathcal{Q}$ is a complete join semilattice (i.e. a partial order with an operation forming joins of arbitrary sets) together with an associative, commutative, binary operation $\otimes$ and constant 1 such that

$$
\begin{aligned}
& q \otimes \mathbf{1}=q \\
& q \otimes \vee P=\vee\{q \otimes p \mid p \in P\}
\end{aligned}
$$

Entailment is interpreted as the order relation, $\leq$, on the underlying lattice of a quantale. The logical operation, $\otimes$, is interpreted by the corresponding binary operation in the quantale and the logical constant $\mathbf{1}$ is interpreted as $\mathbf{1}$ in the quantale. The disjunction, $\oplus$, of linear logic is understood as binary join and the conjunction, \&, as binary meet. The logical constants $\mathrm{T}$ and $\mathrm{F}$ are interpreted as the top and bottom element respectively of the complete lattice. Linear implication is a derived operation:

$$
p \multimap q={ }_{\text {def }} \bigvee\{r \mid r \otimes p \leq q\}
$$

with respect to a quantale. The definition is analogous to that of implication on a complete Heyting algebra, but this time w.r.t. $\otimes$ in place of $\wedge$. The definition of linear implication ensures the adjunction:

$$
r \otimes p \leq q \quad \text { iff } \quad r \leq p \multimap q
$$

With respect to a quantale, and interpretations of the atomic propositions as elements of a quantale, we can inductively associate a proposition $A$ in linear logic with its denotation as a quantale element $\llbracket A \rrbracket$. An entailment

$$
A_{1}, \ldots, A_{n} \models A
$$

\footnotetext{
${ }^{1}$ As originally defined, quantales need not be commutative and should satisfy the idempotency law $q \otimes q=q$. We shall take quantales to be commutative and relax the idempotency law.
} 
holds in the quantale iff

$$
\llbracket A_{1} \rrbracket \otimes \cdots \otimes \llbracket A_{n} \rrbracket \leq \llbracket A \rrbracket .
$$

The special case where $n=0$ is allowed, in which case the situation amounts to

$$
\models A \quad \text { iff } \quad \mathbf{1} \leq \llbracket A \rrbracket .
$$

It is a routine matter to check that each rule is sound with respect to this interpretation. For example the right and left introduction rules for disjunction, $\oplus$, and conjunction, \&, express that they are the join and meet with respect to entailment. In this way it can be seen that, with respect to a quantale:

Theorem 2 If $\vdash A$ then $\models A$.

We have so far ignored the treatment of $! A$. The rules of (1) for $! A$ are interderivable with the following single rule:

$$
\overline{! A \vdash 1 \& A \&(! A \otimes ! A)}
$$

So, as an interpretation of $! q$, for an element $q$ of a quantale, we require an element $x$ such that

$$
x \leq 1 \& q \&(x \otimes x) .
$$

This will not in general characterise a unique value of the quantale; for instance taking $x$ to be the bottom element of the lattice will always do. However from (2) it follows that any $x$ satisfying (3) should be below ! $q$, and hence $! q$ should be the greatest postfixed point, and so fixed point, of

$$
x \mapsto 1 \& q \&(x \otimes x)
$$

in the complete lattice given together with the quantale. Such a solution ensures the soundness of the proof rules extended by those for $! A$.

\section{Petri Nets}

Petri nets are a model of processes (or systems) in terms of types of resources, represented by places which can hold to arbitrary nonnegative 
multiplicity, and how those resources are consumed or produced by actions, represented by transitions. They are described using the notation of multisets.

A multiset over a set $P$ is a function, $M: P \longrightarrow \mathbb{N}$. We shall henceforth only be concerned with finite multisets, i.e. $\{a \in P \mid M(a) \neq 0\}$ finite. With addition, + , of multisets defined by $\left(M+M^{\prime}\right)(a)=M(a)+M^{\prime}(a)$ for all $a \in P$, multisets over $P$ form a (free) commutative monoid with $\underline{0}$ $(\forall a \in P . \underline{0}(a)=0)$, the empty multiset, as unit.

We take a Petri net $N$ to consist of $\left(P, T,{ }^{\bullet}(-),(-)^{\bullet}\right)$, where $P$, a set of places, and $T$, a set of transitions, are accompanied by maps ${ }^{\bullet}(-),(-)^{\bullet}$ on transitions $T$ which for each $t \in T$ give a multiset of $P$, called the pre- and post (multi)set of $t$ respectively. For the moment there are none of the usual restrictions on the net, such as absence of isolated elements, and in particular transitions with empty pre sets and/or post sets will be allowed. And we are actually considering nets with unconstrained capacity.

A Petri net possesses a notion of state, intuitively corresponding to a finite distribution of resources, formalized in the definition of a marking. A marking of $N$ will simply be a finite multiset over $P$. We use $\mathcal{M}$ to denote the set of markings of the net, understood from the context. Sometimes nets are associated with an initial marking $M_{0}$. The behaviour of a net is expressed by saying how markings change as transitions occur (or fire). For markings, $M, M^{\prime}$, and a transition $t \in T, M[t\rangle M^{\prime}$ stands for $t$ fires from $M$ to $M^{\prime}$; that is the firing relation $[t\rangle$ is given by

$$
M[t\rangle M^{\prime} \quad \text { iff } \quad \exists M^{\prime \prime} \in \mathcal{M} \cdot M=M^{\prime \prime}+{ }^{\bullet} t \text { and } t^{\bullet}+M^{\prime \prime}=M^{\prime} .
$$

So $t$ is enabled at $M$ if there is an $M^{\prime} \in \mathcal{M}$ such that $M[t\rangle M^{\prime}$. We shall write $M \rightarrow M^{\prime}$ for the reachability relation, the reflexive and transitive closure of the firing relations. We shall use $\downarrow(M)$ to denote the set of markings which can reach $M$. We will generally call this set the downwards closure of $M$. It is defined by $\downarrow(M)=\left\{M^{\prime} \in \mathcal{M} \mid M^{\prime} \rightarrow M\right\}$.

Petri nets can be presented by using the well-known graphical notation, which we will use in an example. Places are represented by circles, transitions as squares, and arcs of appropriate multiplicities used to indicate the pre and post sets. The formal definitions can then be brought to life in the so called "token game" where markings are visualized as consist- 
ing of a distribution of tokens over places; the number of tokens residing on a place expresses the multiplicity to which it holds according to the marking. The tokens are consumed and produced as transitions occur. A basic reference for Petri nets is [Rei85].

\section{Petri-Net Interpretation}

For simplicity we consider a linear logic language where the atomic propositions are places of nets. I.e. formulae are given by:

$$
\begin{aligned}
& A::=\mathrm{T}|\mathrm{F}| \mathbf{1} \quad \text { constants } \\
& \mid a \quad \text { atoms } \\
& |A \otimes A| A \multimap A \text { multiplicative connectives } \\
& |A \& A| A \oplus A \quad \text { additive connectives } \\
& \mid ! A \quad \text { exponential connective }
\end{aligned}
$$

We make the choice of interpreting an atomic proposition as the downwards closure of the associated place, but we could just as well have used the downwards closure of some marking without altering our results. This choice is consistent with the following intuitive understanding: the denotation of an assertion is to be thought of as the set of requirements sufficient to establish it. This reading will be discussed further shortly, after presenting the semantics. More abstractly, we are giving a semantics in a quantale $\mathcal{Q}$ consisting of downwards-closed subsets of markings with respect to reachability, ordered by inclusion, with a binary operation given by

$$
q_{1} \otimes q_{2}={ }_{\text {def }}\left\{M \mid \exists M_{1} \in q_{1}, M_{2} \in q_{2} . M \rightarrow M_{1}+M_{2}\right\} .
$$

With respect to a net $N$, linear logic formulae are interpreted as follows. The denotation of an assertion can be thought of as consisting of the set of markings which satisfy it. 


$$
\begin{array}{ll}
\llbracket \mathrm{T} \rrbracket_{N} & =\mathcal{M} \\
\llbracket \mathrm{F} \rrbracket_{N} & =\emptyset \\
\llbracket \mathbf{1} \rrbracket_{N} & =\{M \mid M \rightarrow \underline{0}\} \\
\llbracket a \rrbracket_{N} & =\{M \mid M \rightarrow a\} \\
\llbracket A \otimes B \rrbracket_{N} & =\left\{M \mid \exists M_{A} \in \llbracket A \rrbracket_{N}, M_{B} \in \llbracket B \rrbracket_{N} \cdot M \rightarrow M_{A}+M_{B}\right\} \\
\llbracket A \multimap B \rrbracket_{N} & =\left\{M \mid \forall M_{A} \in \llbracket A \rrbracket_{N} \cdot M+M_{A} \in \llbracket B \rrbracket_{N}\right\} \\
\llbracket A \& B \rrbracket_{N} & =\llbracket A \rrbracket_{N} \cap \llbracket B \rrbracket_{N} \\
\llbracket A \oplus B \rrbracket_{N} & =\llbracket A \rrbracket_{N} \cup \llbracket B \rrbracket_{N} \\
\llbracket ! A \rrbracket_{N} & =\bigcup\left\{q \mid q \text { a postfixed point of } x \mapsto \mathbf{1} \cap \llbracket A \rrbracket_{N} \cap(x \otimes x)\right\}
\end{array}
$$

The final clause gives the denotation of $! A$ as a maximum fixed point. The above definitions correspond to the quantale semantics which is determined once we fix the interpretation of atoms (see [EW90]). The semantics of [Bro89] is similar, but somehow dual to that here.

Because of the interpretation of $\mathbf{1}$, validity of an assertion $A$ for the given net, $N$, is defined by

$$
\models_{N} A \quad \text { iff } \quad \underline{0} \in \llbracket A \rrbracket_{N} .
$$

Semantic entailment between assertions $A$ and $B$ is given by

$$
A \models_{N} B \quad \text { iff } \quad \llbracket A \rrbracket_{N} \subseteq \llbracket B \rrbracket_{N} .
$$

Because of the interpretation of linear implication, this is equivalent to

$$
\models_{N} A \multimap B .
$$

For $\Gamma=A_{1}, \ldots, A_{n}$ denote $A_{1} \otimes \cdots \otimes A_{n}$ by $\otimes \Gamma$. We write $\Gamma \models_{N} B$ for $\otimes \Gamma \models{ }_{N} B$.

General validity, $\models A$, of an assertion $A$ is defined by

$$
\models A \quad \text { iff } \quad \models{ }_{N} A, \text { for every net } N
$$

and with respect to entailment: $\Gamma \models B$ iff $\Gamma \models{ }_{N} B$, for every net $N$.

As a special case that quantale semantics is sound, we have the soundness result:

Theorem 3 If $\Gamma \vdash A$ then $\Gamma \models A$. 
So we see that with respect to a Petri net, an assertion $A$ is denoted by a set of markings $\llbracket A \rrbracket_{N}$. As we have discussed, a marking of net can be viewed as a distribution of resources. When $M \in \llbracket A \rrbracket_{N}$ we can think of the marking $M$ as a distribution of resources sufficient to establish $A$ according to the net; in this sense the marking $M$ is one of the (in general many) requirements sufficient to establish $A$. The meaning of an assertion $A$ is specified by saying what requirements are sufficient to establish it - this is the content of the denotation $\llbracket A \rrbracket_{N}$. Accordingly, a net satisfies an assertion $A$ when $\underline{0} \in \llbracket A \rrbracket_{N}$, expressing that $A$ can be established with no resources.

This reading squares with the fact that assertions denote subsets of markings which are downwards closed with respect to the reachability relation of the net; if $M \in \llbracket A \rrbracket_{N}$, so $M$ is a requirement sufficient to establish $A$, and $M^{\prime} \rightarrow M$ so we can obtain $M$ for $M^{\prime}$, then so also is $M^{\prime}$ a sufficient requirement of $A$. Casting an eye over the definition of the semantics of assertions we can read, for example, the definition of $\llbracket a \rrbracket_{N}$, for an atom $a$, as expressing that a sufficient requirement of $a$ is any marking from which the (singleton) marking $a$ can be reached according to the net. Similarly, the sufficient requirements of $A \& B$ are precisely those which are sufficient requirements of both $A$ and of $B$. An element of $\llbracket A \multimap B \rrbracket_{N}$ can be seen as what is required, in addition to any requirement of $A$, in order to establish $B$. There are similar restatements of the semantics for the other connectives as well.

This understanding should be born in mind when considering the example that follows, where we make use of the fact that $\otimes$, \& and $\oplus$ are associative and assume the precedence: $\multimap<\&, \oplus<\otimes$.

Notation: For a multiset, $M$, of assertions of our logic, we associate the formula $\widehat{M}$ which when $M$ is nonempty is given by

$$
\bigotimes_{M(A) \neq 0} A^{M(A)} \quad \text { where } A^{0}=\mathbf{1} \text { and } A^{n}=\overbrace{A \otimes \cdots \otimes A}^{n} \text {, for } n>0
$$

and otherwise, when $M=\underline{0}$, is given by the formula $\mathbf{1}$. We shall not bother to distinguish $M$ and $\widehat{M}$ except for a few crucial statements and proofs.

We can then express that one marking, $M^{\prime}$, is reachable from another $M$ : 
Proposition 4 For any multisets of atoms $M$ and $M^{\prime}$,

$$
M \rightarrow M^{\prime} \text { in the net } N \quad \text { iff } \quad \models_{N} M \multimap M^{\prime} .
$$

Example 5 (Mutual exclusion) Consider the net $N$ :

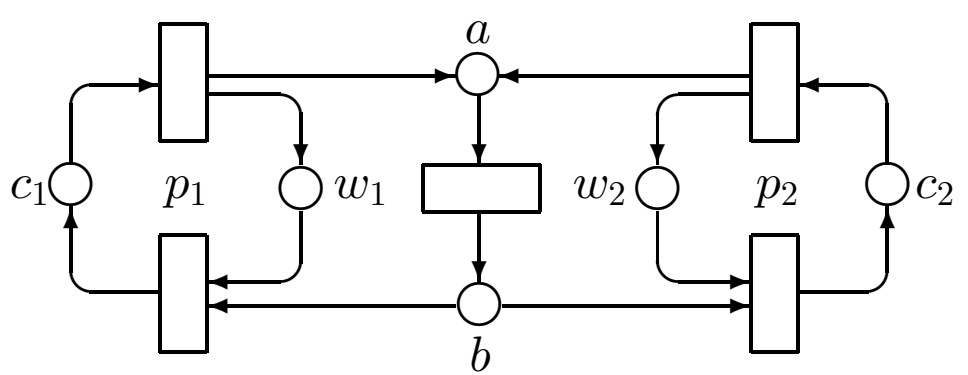

where the marking of the place $w_{1}$ indicates that the first process, $p_{1}$, is working outside its critical region, $c_{1}$, and similarly for the other process, $p_{2}$. The resource corresponding to $b$ is used to ensure mutual exclusion of the critical regions and after a process has been in its critical region it returns a resource, $a$, which then is prepared (transformed into $b$ ) for the next turn. The initial marking, $M_{0}$, will be $M_{0}=b \otimes w_{1} \otimes w_{2}$. We can now express that e.g. $p_{1}$ can enter its critical region (from the initial marking) by: $\models_{N} M_{0} \multimap c_{1} \otimes \mathrm{T}$. However this does not ensure that no undesired tokens are present, so it is better to express it: $\models_{N} M_{0} \multimap c_{1} \otimes w_{2}$. If the system is in a "working state" then both processes have the possibility of entering their critical section: $\models_{N} w_{1} \otimes(a \oplus b) \otimes w_{2} \multimap c_{1} \otimes w_{2} \& w_{1} \otimes c_{2}$. The property, that when $p_{1}$ is in its critical section and $p_{2}$ is working it is possible that $p_{2}$ can later come into its critical section with $p_{1}$ working, is expressed by: $\models_{N} c_{1} \otimes w_{2} \multimap w_{1} \otimes c_{2}$. Similar other "positive" properties can be expressed. Shortly we shall see how to express the "negative" property that both processes cannot be in their critical regions at the same time.

\section{Elementary Completeness Results}

In this section we shall be concerned with completeness results for different fragments of linear logic without exponentials.

We start by sketching the completeness proof for quantale semantics. 
The idea in showing completeness is to build a quantale by taking the ideal completion of the Lindenbaum algebra. More precisely take $Q$ to be $\subseteq$-ordered set of subsets $I$ of assertions of linear logic, without exponentials, such that

$$
\begin{aligned}
& A \vdash B \in I \Rightarrow A \in I, \\
& X \subseteq^{f i n} I \Rightarrow \oplus X \in I
\end{aligned}
$$

(We understand $\oplus \emptyset=\mathrm{F}$ ) .

The $\otimes$-operation on ideals is got by taking:

$$
I \otimes J={ }_{\operatorname{def}}\{C \mid \exists A \in I, B \in J . C \vdash A \otimes B\} .
$$

That this yields an ideal follows routinely: clearly $I \otimes J$ is closed with respect to entailment, i.e. $B \vdash C \in I \otimes J$ implies $B \in I \otimes J$; it is closed under $\oplus$ because it contains $\oplus \emptyset=\mathrm{F}$ and if $C, C^{\prime} \in I \otimes J$ then $C \vdash A \otimes B$ and $C^{\prime} \vdash A^{\prime} \otimes B^{\prime}$, for $A, A^{\prime} \in I, B, B^{\prime} \in J$, whence

$$
\begin{aligned}
C \oplus C^{\prime} & \vdash(A \otimes B) \oplus\left(A^{\prime} \otimes B^{\prime}\right) \\
& \vdash(A \otimes B) \oplus\left(A^{\prime} \otimes B\right) \oplus\left(A \otimes B^{\prime}\right) \oplus\left(A^{\prime} \otimes B^{\prime}\right) \\
& \vdash\left(A \oplus A^{\prime}\right) \otimes\left(B \oplus B^{\prime}\right),
\end{aligned}
$$

where $\left(A \oplus A^{\prime}\right) \in I$ and $\left(B \oplus B^{\prime}\right) \in J$ thus ensuring $C \oplus C^{\prime} \in I \otimes J$ - thus it is closed under $\oplus$.

The quantale $Q$ interprets assertions once we decide to interpret atoms $a$ in the following way:

$$
\llbracket a \rrbracket_{Q}=\{B \mid B \vdash a\} .
$$

It is a relatively simple matter to show the following agreement between the semantics in the constructed quantale and the proof system:

Lemma 6 Letting $A$ be an assertion of linear logic without exponentials,

$$
\llbracket A \rrbracket_{Q}=\{B \mid B \vdash A\} .
$$

Proof By structural induction. We consider two cases:

$A \equiv A_{1} \oplus A_{2}$ : The denotation $\llbracket A_{1} \oplus A_{2} \rrbracket_{Q}=\llbracket A_{1} \rrbracket_{Q} \vee \llbracket A_{2} \rrbracket_{Q}$, the join in $Q$, which contains $A_{1} \oplus A_{2}$, and hence must equal the principal ideal $\left\{B \mid B \vdash A_{1} \oplus A_{2}\right\}$. 
$A \equiv A_{1} \multimap A_{2}$ : By definition,

$$
\llbracket A_{1} \multimap A_{2} \rrbracket_{Q}=\bigvee\left\{I \mid I \otimes \llbracket A_{1} \rrbracket_{Q} \subseteq \llbracket A_{2} \rrbracket_{Q}\right\}
$$

a join in $Q$ which contains $A_{1} \multimap A_{2}$ and hence includes the principal ideal $\left\{B \mid B \vdash A_{1} \multimap A_{2}\right\}$. It is in fact equal to this principal ideal. To see this, let $B \in I$ where $I \otimes \llbracket A_{1} \rrbracket_{Q} \subseteq \llbracket A_{2} \rrbracket_{Q}$. Then $B \otimes A_{1} \in \llbracket A_{2} \rrbracket_{Q}$, so by structural induction $B \otimes A_{1} \vdash A_{2}$, whence $B \vdash A_{1} \multimap A_{2}$.

Corollary 7 Let $A$ be an assertion of linear logic without exponentials. Then

$$
\models_{Q} A \quad \text { iff } \quad \vdash A \text {. }
$$

As a corollary we from $A \vdash B$ iff $\vdash A \multimap B$ obtain completeness with respect to quantales:

Theorem 8 For the fragment without exponentials we have:

$$
\Gamma \models A \quad \text { iff } \quad \Gamma \vdash A .
$$

In the remaining sections we shall only be concerned with completeness proofs for net semantics.

\subsection{Completeness for the $\oplus$-free Fragment}

Restrict the syntax to the fragment:

$$
A::=\mathrm{\top}|\mathbf{1}| a\left|A_{1} \& A_{2}\right| A_{1} \otimes A_{2} \mid A_{1} \multimap A_{2}
$$

where $a$ ranges over atoms. For the $\oplus$-free fragment we construct a net $N$ where the places are formulae and the transitions essentially correspond to the provable sequents. I.e.

- Places are assertions of $(\oplus$-free) above.

- Transitions are pairs $\left(M, M^{\prime}\right)$ of multisets of places for which $\widehat{M} \vdash$ $\widehat{M}^{\prime}$ with pre- and postset maps $\bullet\left(M, M^{\prime}\right)=M$ and $\left(M, M^{\prime}\right)^{\bullet}=M^{\prime}$. 
Lemma 9 For markings $M, M^{\prime}$ of the net $N$,

$$
M \rightarrow M^{\prime} \text { in the net } \quad \text { iff } \quad \widehat{M} \vdash \widehat{M}^{\prime} .
$$

Proof "if": It is clear by definition that if $\widehat{M} \vdash \widehat{M^{\prime}}$ then $M \rightarrow M^{\prime}$ for any markings $M, M^{\prime} \in \mathcal{M}$.

"only if": Follows by a simple inductive argument once we have established

$$
M[t\rangle M^{\prime} \text { implies } \widehat{M} \vdash \widehat{M^{\prime}} .
$$

However, if $M[t\rangle M^{\prime}$ then, by definition, there is some $M^{\prime \prime} \in \mathcal{M}$ such that

$$
M=M^{\prime \prime}+{ }^{\bullet} t \text { and } t^{\bullet}+M^{\prime \prime}=M^{\prime} .
$$

From $\widehat{\bullet} \vdash \widehat{t^{\bullet}}$ we derive $\widehat{M^{\prime \prime}} \otimes \widehat{\bullet} \vdash \widehat{t^{\bullet}} \otimes \widehat{M^{\prime \prime}}$. The result then follows from $\widehat{M} \dashv \vdash \widehat{M^{\prime \prime}} \otimes \widehat{\bullet}$ and $\widehat{M^{\prime}} \dashv-\widehat{t^{\bullet}} \otimes \widehat{M^{\prime \prime}}$.

Lemma 10 For the $\oplus$-free fragment we have: $\llbracket A \rrbracket_{N}=\{M \mid \widehat{M} \vdash A\}$.

Proof By induction on the structure of $A$ using the previous lemma.

$A \equiv \mathrm{T}: \llbracket \mathrm{T} \rrbracket_{N}=\mathcal{M}=\{M \in \mathcal{M} \mid M \vdash \mathrm{T}\}$ by axiom $\Gamma \vdash \mathrm{T}$ (recall $\mathcal{M}$ consists of finite multisets).

$A \equiv \mathbf{1}: \llbracket \mathbb{1} \rrbracket_{N}=\{M \in \mathcal{M} \mid M \rightarrow \underline{0}\}=\{M \in \mathcal{M} \mid M \vdash \underline{\hat{0}}=\mathbf{1}\}$ by lemma 9 .

$A \equiv a: \llbracket a \rrbracket_{N}=\{M \in \mathcal{M} \mid M \rightarrow a\}=\{M \in \mathcal{M} \mid M \vdash a\}$ by lemma 9.

$A \equiv A_{1} \otimes A_{2}$ :

$M \in \llbracket A_{1} \otimes A_{2} \rrbracket_{N}$

$\Leftrightarrow \exists M_{1} \in \llbracket A_{1} \rrbracket_{N}, M_{2} \in \llbracket A_{2} \rrbracket_{N} . M \rightarrow M_{1}+M_{2}$ by definition,

$\Leftrightarrow \exists M_{1}, M_{2} \in \mathcal{M} . M_{1} \vdash A_{1}, M_{2} \vdash A_{2}$ and $M \rightarrow M_{1}+M_{2}$ by induction,

$\Leftrightarrow \exists M_{1}, M_{2} \in \mathcal{M} . M_{1} \vdash A_{1}, M_{2} \vdash A_{2}$ and $M \vdash M_{1} \otimes M_{2}$ by lemma 9 ,

$\Leftrightarrow M \vdash A_{1} \otimes A_{2}$ by $(\vdash \otimes),(\otimes \vdash)$ and (identity).

$A \equiv A_{1} \multimap A_{2}$ :

$M \in \llbracket A_{1} \multimap A_{2} \rrbracket_{N} \Leftrightarrow \forall M_{1} \in \llbracket A_{1} \rrbracket_{N} . M+M_{1} \in \llbracket A_{2} \rrbracket_{N}$ by definition,

$\Leftrightarrow \forall M_{1} \in \mathcal{M} . M_{1} \vdash A_{1} \Rightarrow M \otimes M_{1} \vdash A_{2}$ by induction,

$\Rightarrow M \vdash A_{1} \multimap A_{2}$ by $(\vdash \multimap)$ and (identity).

To see " $\Leftarrow$ " suppose $M \vdash A_{1} \multimap A_{2}$. From $(\multimap \vdash)$ we derive $M, A_{1} \vdash A_{2}$. Using (cut) and $M_{1} \vdash A_{1}$ we then get $M, M_{1} \vdash A_{2}$. 
$A \equiv A_{1} \& A_{2}:$

$M \in \llbracket A_{1} \& A_{2} \rrbracket_{N} \Leftrightarrow M \in \llbracket A_{1} \rrbracket_{N}$ and $M \in \llbracket A_{2} \rrbracket_{N}$ by definition,

$\Leftrightarrow M \vdash A_{1}$ and $M \vdash A_{2}$ by induction,

$\Rightarrow M \vdash A_{1} \& A_{2}$ by $(\vdash \&)$.

For the other direction " $\Leftarrow$ " we by (identity) and $(l \& \vdash)$ obtain $A_{1} \& A_{2} \vdash$ $A_{1}$ and so $M \vdash A_{1}$ from $M \vdash A_{1} \& A_{2}$ and (cut). By symmetry $M \vdash A_{2}$.

Because $\models_{N} A$ follows from $\models A$, and the fragment contains implication we deduce:

Theorem 11 For the $\oplus$-free fragment we have:

$$
\Gamma \models A \quad \text { iff } \quad \Gamma \vdash A .
$$

As observed by Sergei Soloviev, the net need for a particular sequent only to be constructed with a finite number of places corresponding to subformulae of the sequent. However, it not clear that the net can be finite if the sequent contains \& or $\multimap$.

\subsection{Completeness for the - -free Fragment}

We can obtain completeness for the - -free fragment of propositional intuitionistic logic. Its syntax:

$$
A::=\mathrm{T}|\mathrm{F}| \mathbf{1}|a| A_{1} \oplus A_{2}\left|A_{1} \& A_{2}\right| A_{1} \otimes A_{2} \quad \text { ( } \circ \text {-free) }
$$

where $a$ ranges over atoms. With a similar construction to that in the previous section we can obtain a rather weak form of completeness for the $\multimap$-free fragment.

Lemma 12 For the $\multimap$-free fragment we have $\llbracket A \rrbracket_{N} \subseteq\{M \mid M \vdash A\}$.

Proof Induction on the structure of $A$. All the cases except $A \equiv \mathrm{F}$ and $A \equiv A_{1} \oplus A_{2}$ are handled exactly as the $\subseteq$-part of lemma 10 (notice the weaker hypothesis).

$A \equiv \mathrm{F}:$ Evident as $\llbracket \mathrm{F} \rrbracket_{N}=\emptyset$. 
$A \equiv A_{1} \oplus A_{2}:$

$M \in \llbracket A_{1} \oplus A_{2} \rrbracket_{N}$

$\Leftrightarrow M \in \llbracket A_{1} \rrbracket_{N}$ or $M \in \llbracket A_{2} \rrbracket_{N}$ by definition,

$\Rightarrow M \vdash A_{1}$ or $M \vdash A_{2}$ by induction,

$\Rightarrow M \vdash A_{1} \oplus A_{2}$ by $(\vdash \oplus l)$ or $(\vdash \oplus r)$.

As a corollary we have:

Theorem 13 For the - -free fragment we have:

$$
\models A \quad \text { iff } \quad \vdash A .
$$

We have not used the distributive law yielded by the net semantics:

$$
(A \oplus B) \& C \vdash(A \& C) \oplus(B \& C)
$$

(\&- $\oplus$-dist. $)$

With this as an additional proof rule we can obtain a stronger completeness result for the $\multimap$-free fragment of propositional intuitionistic logic.

To show completeness we construct a net with places (and markings) identified with assertions in the $\oplus$-free subfragment:

$$
A::=\mathrm{\top}|\mathbf{1}| a\left|A_{1} \& A_{2}\right| A_{1} \otimes A_{2}
$$

We will just call it the $\oplus$-free fragment in the rest of this section. Construct a net $N$ where:

- Places are assertions in the $\oplus$-free fragment.

- Transitions are pairs $\left(M, M^{\prime}\right)$ of multisets of places for which $\widehat{M} \vdash \widehat{M}^{\prime}$.

Lemma 14 For markings $M, M^{\prime}$ of the net, $M \rightarrow M^{\prime}$ in the net $\quad$ iff $\quad M, M^{\prime} \oplus$-free and $M \vdash M^{\prime}$ in the logic.

Proof The proof is like that for lemma 9.

Lemma 15 (Decomposition lemma). For any $\multimap$-free assertion $A$ there is a finite set $I$ indexing $\multimap-\oplus$-free assertions $M_{i}$, such that

$$
A \dashv \vdash \bigoplus_{i \in I} M_{i} .
$$


Proof The proof proceeds by structural induction on the assertion $A$. The base cases are routine; for example $F \dashv \mapsto \emptyset$ (=F by definition), i.e. falsity is interderivable with the empty disjunction. Of the remaining cases, that where $A$ has the form $A_{1} \& A_{2}$ makes use, as is to be expected, of the additional distributivity rules for $\&$ and $\oplus$. Inductively, assume

$$
A_{1} \dashv \vdash \bigoplus_{i \in I} M_{i}^{1} \text { and } A_{2} \dashv \Vdash \bigoplus_{j \in J} M_{j}^{2} \text {. }
$$

Then, from these assumptions and repeated use of $\&$ - $\oplus$-distributively

$$
\begin{aligned}
A_{1} \& A_{2} & \dashv\left(\oplus_{i} M_{i}^{1}\right) \&\left(\oplus_{j} M_{j}^{2}\right) \\
& \dashv \oplus_{i}\left(M_{i}^{1} \&\left(\oplus_{j} M_{j}^{2}\right)\right) \\
& -\Vdash \oplus_{(i, j) \in I \times J}\left(M_{i}^{1} \& M_{j}^{2}\right) .
\end{aligned}
$$

The case where $A$ has the form $A_{1} \otimes A_{2}$ is exactly analogous, making use instead of the standard $\otimes$ - $\oplus$-distributivity of linear logic.

Lemma 16 Let $\Gamma=B_{1}, \ldots, B_{n}$, possibly empty, be list of assumptions in the $\oplus$-free fragment above. Then,

$$
\Gamma \nvdash \mathrm{F}
$$

and

$$
\text { if } \Gamma \vdash C \oplus D \text { then } \Gamma \vdash C \text { or } \Gamma \vdash D \text {. }
$$

Proof By cut-elimination any proof of a sequent can be replaced by a cut-free proof. The above lemma follows by induction or the size of cut-free proofs.

Lemma 17 For any $\multimap$-free assertion $A$,

$$
\llbracket A \rrbracket_{N}=\{M \mid M \text { is } \oplus \text {-free, } M \vdash A\} .
$$

Proof The proof proceeds by structural induction on the assertion $A$. $A \equiv \mathrm{T}$ : Clearly, $\llbracket \mathrm{T} \rrbracket_{N}=\mathcal{M}=\{M \mid M \oplus$-free $\}=\{M \oplus$-free $\mid M \vdash \mathrm{T}\}$. $A \equiv \mathrm{F}:$ Now, using lemma $16, \llbracket \mathrm{F} \rrbracket_{N}=\emptyset=\{M \mid M \oplus$-free, $\vdash \mathrm{F}\}$. 
$A \equiv \mathbf{1}: \llbracket \mathbf{1} \rrbracket_{N}=\{M \mid M \rightarrow \underline{0}\}=\{M \mid M \oplus$-free, $M \vdash \mathbf{1}\}$ by lemma 14 .

$A \equiv a: \llbracket a \rrbracket_{N}=\{M \mid M \rightarrow a\}=\{M \mid M \oplus$-free, $M \vdash a\}$ by lemma 14 .

$A \equiv A_{1} \& A_{2}:$ We argue straightforwardly that

$M \in \llbracket A_{1} \& A_{2} \rrbracket_{N} \Leftrightarrow M \in \llbracket A_{1} \rrbracket_{N}$ and $M \in \llbracket A_{2} \rrbracket_{N}$ by definition,

$\Leftrightarrow M \oplus$-free, $M \vdash A_{1}$ and $M \vdash A_{2}$ by induction,

$\Leftrightarrow M \oplus$-free, $M \vdash A_{1} \& A_{2}$ by the proof rules.

$A \equiv A_{1} \oplus A_{2}$ : Argue:

$M \in \llbracket A_{1} \oplus A_{2} \rrbracket_{N}$

$\Leftrightarrow M \in \llbracket A_{1} \rrbracket_{N}$ or $M \in \llbracket A_{2} \rrbracket_{N}$ by definition,

$\Leftrightarrow M \oplus$-free and either $M \vdash A_{1}$ or $M \vdash A_{2}$ by induction,

$\Leftrightarrow M \oplus$-free, $M \vdash A_{1} \oplus A_{2}$ by lemma 16 and $(\vdash \oplus)$.

$A \equiv A_{1} \otimes A_{2}$ : The proof in this case is a little more involved. Argue:

$M \in \llbracket A_{1} \otimes A_{2} \rrbracket_{N}$

$\Leftrightarrow \exists M_{1} \in \llbracket A_{1} \rrbracket_{N}, M_{2} \in \llbracket A_{2} \rrbracket_{N} . M \rightarrow M_{1}+M_{2}$ by definition,

$\Leftrightarrow M \oplus$-free, $\exists M_{1}, M_{1} \oplus$-free. $M_{1} \vdash A_{1}, M_{2} \vdash A_{2}$ and

$M \vdash M_{1} \otimes M_{2}$ by induction and lemma 14 ,

$\Rightarrow M \oplus$-free, $M \vdash A_{1} \otimes A_{2}$ from the proof rules.

To show the converse implication, and so equivalence, assume $M$ is $\oplus$-free and $M \vdash A_{1} \otimes A_{2}$. By lemma 15, we may assume

$$
A_{1} \dashv \Vdash \bigoplus_{i \in I} M_{i}^{1} \text { and } A_{2} \dashv \Vdash \bigoplus_{j \in J} M_{j}^{2} .
$$

We may furthermore assume $I$ and $J$ to be nonempty. Otherwise $A_{1} \otimes$ $A_{2} \dashv \vdash \mathrm{F}$ and so, as $M$ is $\oplus$-free, $M \nvdash A_{1} \otimes A_{2}$ by lemma 16-a contradiction.

Therefore

$$
M \vdash\left(\bigoplus_{i \in I} M_{i}^{1}\right) \otimes\left(\bigoplus_{j \in J} M_{j}^{2}\right),
$$

so by distributivity,

$$
M \vdash \bigoplus_{(i, j) \in I \times J} M_{i}^{1} \otimes M_{j}^{2}
$$

Hence, by lemma 16,

$$
M \vdash M_{i}^{1} \otimes M_{j}^{2} \text { for some } i \in I, j \in J
$$

such that $M_{i}^{1} \vdash A_{1}$ and $M_{j}^{2} \vdash A_{2}$. This plainly gives the required converse. 
Corollary $18 \models_{N} A$ iff $\vdash A$, for any $\multimap$-free assertion $A$.

Thus we have completeness.

Because we only use the decomposition lemma (lemma 15) for the $\otimes$ case of the structural induction in lemma 17, we also get completeness for the larger fragment of assertions $B$ given by:

$$
B::=A|A \multimap B| \mathrm{T}|\mathrm{F}| \mathbf{1}|a| B_{1} \& B_{2} \mid B_{1} \oplus B_{2}
$$

where $A$ lie in the $\multimap$-free fragment and $a$, as usual, ranges over atoms.

Lemma 19 For the larger fragment,

$$
\llbracket B \rrbracket_{N}=\{M \mid M \oplus \text {-free, } M \vdash B\} .
$$

Proof The proof proceeds by structural induction, as in lemma 17, but for a new case where the assertion has the form $A \multimap B$. Because of its assumed form, by lemma 15, there is a decomposition

$$
A \dashv \vdash \bigoplus_{i \in I} M_{i} .
$$

Now, for $\oplus$-free $M$ we argue that

$$
M \in \llbracket A \multimap B \rrbracket_{N}
$$

$\Leftrightarrow \forall M_{A} \in \llbracket A \rrbracket_{N} . M+M_{A} \in \llbracket B \rrbracket_{N}$ by definition,

$\Leftrightarrow \forall M_{A} \oplus$-free. $M_{A} \vdash A \Rightarrow M \otimes M_{A} \vdash B$ by induction,

$\Leftrightarrow \forall M_{A} \oplus$-free. $M_{A} \vdash \oplus_{i \in I} M_{i} \Rightarrow M \otimes M_{A} \vdash B$

$\Leftrightarrow \forall M_{A} \oplus$-free. $\left(\exists i \in I . M_{A} \vdash M_{i}\right) \Rightarrow M \otimes M_{A} \vdash B$ by lemma 16 ,

$\Leftrightarrow \forall i \in I, \forall M_{A} \oplus$-free. $M_{A} \vdash M_{i} \Rightarrow M \otimes M_{A} \vdash B$

$\Leftrightarrow \forall i \in I . M \otimes M_{i} \vdash B$.

Here " $\Rightarrow$ " follows directly by taking $M_{A}=M_{i}$. The converse " $\Leftarrow$ " makes use of the fact that if $M_{A} \vdash M_{i}$ and $M \otimes M_{i} \vdash B$ then $M \otimes M_{A} \vdash B$.

Now, continuing the argument,

$$
\begin{aligned}
M \in \llbracket A \multimap B \rrbracket_{N} & \Leftrightarrow \forall i \in I . M \oplus M_{i} \vdash B \\
& \Leftrightarrow \bigoplus_{i \in I}\left(M \otimes M_{i}\right) \vdash B \text { from the proof system, } \\
& \Leftrightarrow M \otimes\left(\oplus_{i \in I} M_{i}\right) \vdash B \\
& \Leftrightarrow M \otimes A \vdash B \\
& \Leftrightarrow M \vdash A \multimap B .
\end{aligned}
$$


Corollary 20 For the larger fragment, $\models B$ iff $\vdash B$ with the additional $\&$ - $\oplus$-distributivity law.

Theorem 21 For the $\multimap$-free fragment,

$$
\Gamma \models A \quad \text { iff } \quad \Gamma \vdash A
$$

with the additional \&- $\oplus$-distributive law.

Proof Corollary 20 gives

$$
\models \otimes \Gamma \multimap A \text { iff } \vdash \otimes \Gamma \multimap A .
$$

Hence

$$
\Gamma \models A \text { iff } \Gamma \vdash A \text {. }
$$

\section{Quantification and Atomic Nets}

Definition $22 A$ net is atomic iff whenever $M \rightarrow \underline{0}$ then $\underline{0} \rightarrow M$, for any marking $M$.

This corresponds to $\mathbf{1}$ being atomic in the associated quantale - see the remark following the semantics of linear logic formulae in a net.

An interesting consequence of dealing with an atomic net $N$ is, that whatever property we could state before in terms of validity of a closed formula $A$, can now be stated negatively as $\models_{N} A \& \mathbf{1} \multimap \mathrm{F}$. Precisely:

Proposition 23 For an atomic net $N$ and a closed formula $A$,

$$
\models_{N} A \& \mathbf{1} \multimap \mathrm{F} \quad \text { iff } \quad \nvdash_{N} A .
$$

Proof Notice that due to atomicty, the the denotation of $A \& \mathbf{1}$ is that of $\mathbf{1}$ if the denoation of $A$ contains 1 and empty otherwise. Hence $\llbracket A \& \mathbf{1} \multimap \mathrm{F} \rrbracket_{N}$ equals $\mathrm{T}$ in case $\not \models_{N} A$ and equals $\mathrm{F}$ in case $\models_{N} A$.

Abbreviating $A \& \mathbf{1} \multimap \mathrm{F}$ by $\sim A$ and combining this proposition with proposition 4 we can express that a marking $M^{\prime}$ cannot be reached from another $M$ : 
Corollary 24 For multiset of atoms $M$ and $M^{\prime}$,

$$
M \nrightarrow M^{\prime} \text { in an atomic net } N \quad \text { iff } \quad \models_{N} \sim\left(M \multimap M^{\prime}\right) \text {. }
$$

Example 5 (continued)

We can now express that the processes, $p_{1}$ and $p_{2}$ cannot get into their critical regions at the same time. We might try $\models_{N} \sim\left(M_{0} \multimap c_{1} \otimes c_{2}\right)$. This is not quite right however, since $\models_{N} \sim\left(M_{0} \multimap c_{1} \otimes c_{2}\right)$ merely states that the two processes cannot be in their critical regions at the same time when no other tokens are present; the correct statement is $\models_{N} \sim\left(M_{0} \multimap c_{1} \otimes c_{2} \otimes \mathrm{T}\right)$.

We are also able to express that a finite net $N$ is 1-safe by $\models_{N} \sim\left(M_{0} \multimap\right.$ $\left.\left(\oplus_{a \in P} a \otimes a\right) \otimes \mathrm{T}\right)$. That a transition $t$ is $M$-dead in a net $N$, i.e. $\forall M^{\prime} \in$ $[M\rangle . M^{\prime}[t\rangle$, is expressed by

$$
\models_{N} \sim(M \multimap \bullet \otimes \mathrm{T}) .
$$

Notice that $A \& 1$ plays the role of the exponential $! A$, and indeed according the net semantics, when the net $N$ is atomic

$$
\llbracket ! A \rrbracket_{N}=\llbracket A \& \mathbf{1} \rrbracket_{N} .
$$

\section{Syntax}

Assume a countable set of atoms. Define the assertions over the atoms to be:

$$
A::=\mathrm{T}|\mathrm{F}| \mathbf{1}|a| x\left|A_{1} \otimes A_{2}\right| A_{1} \multimap A_{2}\left|A_{1} \& A_{2}\right| A_{1} \oplus A_{2}|\underset{x}{\bigoplus} A| \boldsymbol{\&}_{x} A
$$

where $a$ ranges over the atoms and $x$ ranges over countably many variables. The new constructions $\oplus_{x} A$ and $\&_{x} A$ are forms of existential and universal quantification and bind accordingly. We adopt the traditional notions of free and bound variable and in particular use $\mathrm{FV}(A)$ for the set of free variables in $A$, and more generally $\mathrm{FV}\left(A_{1}, \ldots, A_{n}\right)$ for $\mathrm{FV}\left(A_{1}\right) \cup \cdots \cup \mathrm{FV}\left(A_{n}\right)$. The variables $x$ are to be thought of as standing for markings of a net. 


\section{Semantics}

Given a net $N$, with markings (i.e. finite multisets of places) $\mathcal{M}$, a (marking) environment is a function $\rho$ from variables to markings $\mathcal{M}$. Because of the presence of free variables we define the meaning of an assertion with respect to a marking environment. In particular,

$$
\begin{aligned}
& \llbracket \oplus_{x} A \rrbracket_{N} \rho=\cup_{M \in \mathcal{M}} \llbracket A \rrbracket_{N} \rho[M / x], \\
& \llbracket \&_{x} A \rrbracket_{N} \rho=\cap_{M \in \mathcal{M} \llbracket A \rrbracket_{N} \rho[M / x],} \\
& \llbracket x \rrbracket_{N} \rho=\{M \in \mathcal{M} \mid M \rightarrow \rho(x)\} .
\end{aligned}
$$

Atoms are interpreted as places of the net as in section 5 and similarly validity of a closed assertion $A$ for the given net, $N$, can be expressed by:

$$
\models_{N} A \quad \text { iff } \quad \underline{0} \in \llbracket A \rrbracket_{N} \rho .
$$

This is generalized to open terms by taking the universal closure:

$$
\models_{N} A \quad \text { iff } \quad \underline{0} \in \llbracket \boldsymbol{\&}_{x_{1}} \cdots \boldsymbol{\&}_{x_{k}} A \rrbracket_{N} \rho
$$

where $A$ has free variables $x_{1}, \ldots, x_{k}$ (here $\rho$ can be arbitrary because $\&_{x_{1}} \cdots \&_{x_{k}} A$ is closed).

Let $T$ be a subset of closed assertions in the original syntax. Define

$$
\begin{gathered}
B_{1}, \ldots, B_{n} \models_{T} A \text { iff for all atomic nets } N \text { such that }\left(\forall B \in T . \models_{N} B\right), \\
\models_{N}\left(B_{1} \otimes \cdots \otimes B_{n} \multimap A\right) .
\end{gathered}
$$

Before proceeding with the proof rules we show how the new constructions can be used to express liveness.

A transition $t$ is life iff $\forall M \in\left[M_{0}\right\rangle \exists M^{\prime} \in[M\rangle . M^{\prime}[t\rangle$.

This can be expressed by:

$$
\models_{N} \underset{x}{\mathbb{\&}}\left(\left(M_{0} \multimap x\right) \& \mathbf{1} \multimap(x \multimap \bullet \bullet \otimes T)\right) .
$$

Obviously liveness can then be expressed for finite nets. 


\section{Proof rules}

The proof rules are those of section 2 (without exponentials - they will become definable in the purely propositional logic), together with:

$$
\frac{\Gamma \vdash A}{\Gamma[\theta] \vdash A[\theta]}
$$

where $\theta$ is a substitution of marking terms (i.e. assertions built up from variables, atoms and $\mathbf{1}$ purely by $\otimes$ ) - the usual care to avoid capture of free variables applies here.

$$
\begin{gathered}
\frac{\Gamma, B \vdash A}{\Gamma, \oplus_{x} B \vdash A} \quad x \notin \operatorname{FV}(\Gamma, A) \\
\frac{\Gamma \vdash A[M / x]}{\Gamma \vdash \oplus_{x} A} \text { where } M \text { is a marking term }
\end{gathered}
$$

Note these rules yield (and in the presence of (Subst.) are equivalent with)

$$
\frac{\Gamma, \oplus_{x} B \vdash A}{\Gamma, B \vdash A} \quad x \notin \mathrm{FV}(\Gamma, A)
$$

Assume $(\oplus \vdash)$ and $(\vdash \oplus)$. The upwards direction of the rule ( $\oplus$-adj.) is simply $(\oplus \vdash)$.

The downwards direction viz.

$$
\frac{\Gamma, \oplus_{x} B \vdash A}{\Gamma, B \vdash A} \quad x \notin \operatorname{FV}(\Gamma, A)
$$

is derivable in the following way. Clearly $B \vdash B$ so by application of $(\vdash \oplus), B \vdash \oplus_{x} B$. Now by the cut rule from the assumption $\Gamma, \oplus_{x} B \vdash A$ we can conclude $\Gamma, B \vdash A$.

By using (Subst.) we can also derive $(\oplus \vdash)$ and $(\vdash \oplus)$ from $(\oplus$-adj.). The rule $(\oplus \vdash)$ is simply the upwards reading of $(\oplus$-adj.). Now we show $(\vdash \oplus)$ follows from ( $\oplus$-adj.): Clearly $\oplus_{x} A \vdash \oplus_{x} A$, from which $A \vdash \oplus_{x} A$ follows by $\left(\oplus\right.$-adj.); hence by (Subst.) $A[M / x] \vdash \oplus_{x} A$, making $(\vdash \oplus)$ derivable.

$$
\frac{\Gamma, B \vdash A}{\Gamma, \mathbb{\&}_{x} B \vdash A}
$$




$$
\frac{\Gamma \vdash A}{\Gamma \vdash \&_{x} A} \quad x \notin \mathrm{FV}(\Gamma)
$$

Note these rules are equivalent with

$$
\frac{\Gamma \vdash A}{\overline{\Gamma \vdash \&_{x} A}} \quad x \notin \mathrm{FV}(\Gamma) .
$$

In addition we have the following axioms valid of nets:

$$
\begin{gathered}
\left(A_{1} \oplus A_{2}\right) \& B \vdash\left(A_{1} \& B\right) \oplus\left(A_{2} \& B\right) \\
\left(\bigoplus_{x} A\right) \& B \vdash \bigoplus_{x}(A \& B) \text { where } x \notin \mathrm{FV}(B)
\end{gathered}
$$

In fact, in the presence of the atomicity, basis and primeness axioms, these distributivity laws are derivable from those in the special case where $B$ is $\mathbf{1}$. The other distributive law,

$$
\boldsymbol{\&}_{x}(A \oplus B) \vdash\left(\boldsymbol{\&}_{x} A\right) \oplus B \quad \text { where } x \notin \mathrm{FV}(B),
$$

is also derivable (for general $B$ ).

$$
\vdash\left(\boldsymbol{\&}_{x} B\right) \oplus \bigoplus_{x}((B \& \mathbf{1}) \multimap \mathbf{F})
$$

These entail sequents of the following form (by taking the variable $x$ to not appear in $B$ ):

$$
\vdash B \oplus((B \& \mathbf{1}) \multimap \mathbf{F}) .
$$

These hold because in an atomic net the denotation of a formula $B \& \mathbf{1}$, in an environment for its free variables, only has two possibilities, to be the denotation of $\mathrm{F}$ or the denotation of $\mathbf{1}$.

$$
A \vdash \bigoplus_{x} x \otimes((x \multimap A) \& \mathbf{1}) \quad \text { where } x \notin \mathrm{FV}(A)
$$

These hold in an atomic net because there an assertion is denoted by a set of markings; notice how the expression $(x \multimap A) \& \mathbf{1}$ is equivalent to $\mathbf{1}$ in the case the marking $x$ satisfies $A$ and $\mathrm{F}$ otherwise, so the effect in the whole expression is only to make a contribution of $x$ when this satisfies A. 


$$
\begin{aligned}
(x \multimap \mathrm{F}) & \vdash \mathrm{F} \\
(x \multimap B \oplus C) & \vdash(x \multimap B) \oplus(x \multimap C) \\
\left(x \multimap \oplus_{y} A\right) & \vdash \oplus_{y}(x \multimap A) \text { where } y \text { and } x \text { are distinct. }
\end{aligned}
$$

These axioms hold because if a marking is contained in union, denoting a disjunction, then it is clearly in a component of the union.

For clarity we have collected the new proof rules:

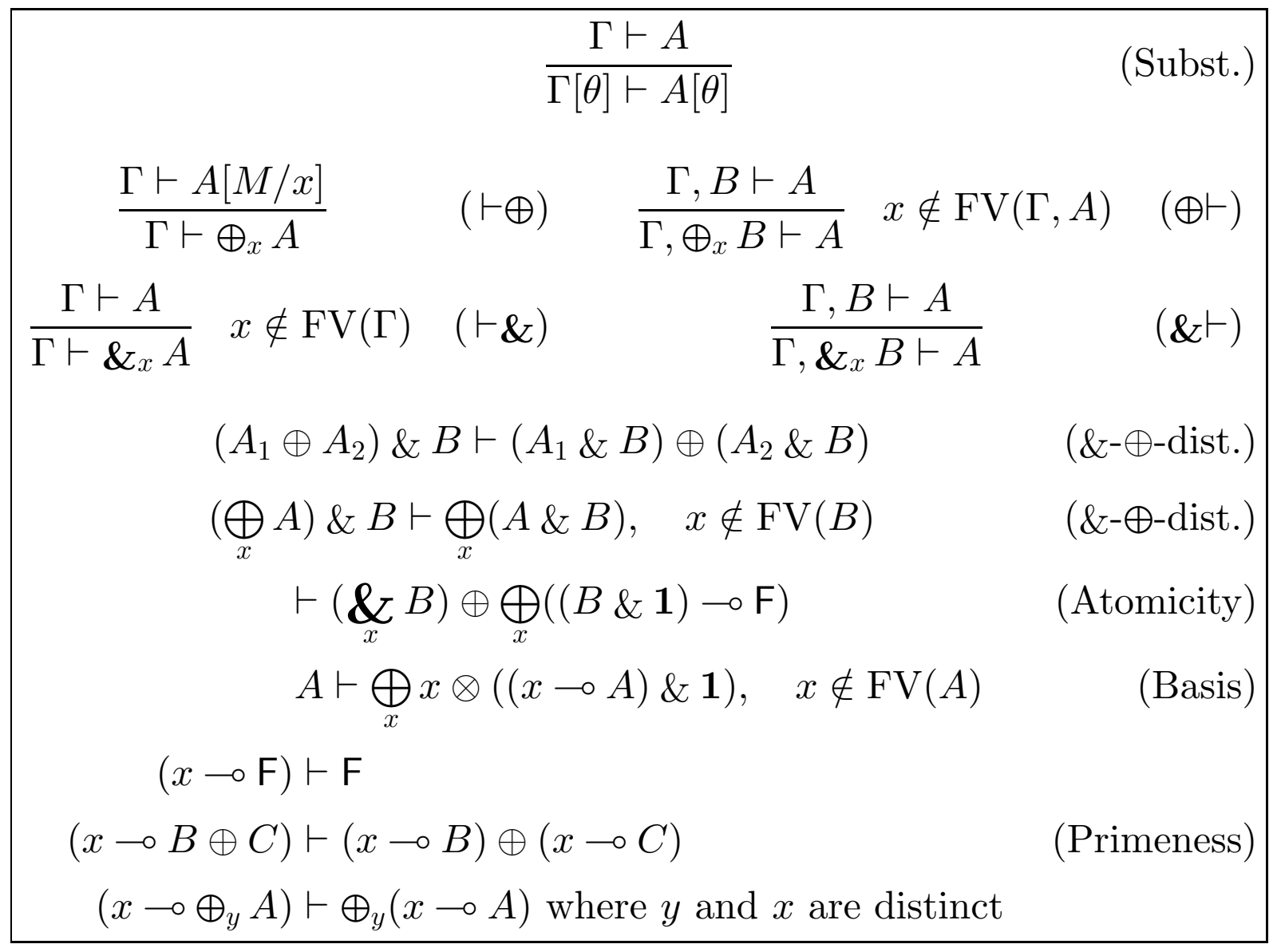

The soundness of the basis and atomicity axioms follows from the fact that, in an atomic net,

$\llbracket A \& \mathbf{1} \rrbracket_{N} \rho=\left\{\begin{array}{l}\mathbf{1} \text { if } \mathbf{1} \subseteq \llbracket A \rrbracket_{N} \rho \\ \mathrm{F} \text { otherwise }\end{array}\right.$ and $\llbracket A \& \mathbf{1} \multimap \mathrm{F} \rrbracket_{N} \rho=\left\{\begin{array}{l}\mathrm{F} \text { if } \mathbf{1} \subseteq \llbracket A \rrbracket_{N} \rho \\ \mathrm{T} \text { otherwise }\end{array}\right.$

We have already remarked that in an atomic net, an exponential $! A$ is represented by $A \& \mathbf{1}$. In fact from the atomicity axioms and the rules for exponentials, there is a fairly direct proof of their equivalence, yielding

$$
! A \dashv A \& 1
$$


- the syntax of exponentials can be eliminated in favour of the purely propositional connectives.

For the proof first remark that $A \& \mathbf{1} \vdash A$ and $A \& \mathbf{1} \vdash \mathbf{1}$, simply by the rules for $\&$. From $A \& \mathbf{1} \vdash(A \& \mathbf{1}) \& \mathbf{1}$ and (Atomicity) we also get $\vdash(A \& \mathbf{1}) \oplus(A \& \mathbf{1} \multimap \mathbf{F})$. Using $A \& \mathbf{1} \vdash A \& \mathbf{1}$ we then deduce

$$
A \& \mathbf{1} \vdash((A \& \mathbf{1}) \otimes(A \& \mathbf{1})) \oplus((A \& \mathbf{1}) \otimes(A \& \mathbf{1} \multimap \mathrm{F}))
$$

so that $A \& \mathbf{1} \vdash((A \& \mathbf{1}) \otimes(A \& \mathbf{1})) \oplus \mathrm{F}$, i.e.

$$
A \& \mathbf{1} \vdash(A \& \mathbf{1}) \otimes(A \& \mathbf{1}) \text {. }
$$

By (2) this ensures $A \& \mathbf{1} \vdash ! A$, and clearly $! A \vdash A \& \mathbf{1}$, making (*).

In constructing prime theories we follow Henkin and extend the original syntax to include new atoms drawn from

$$
c_{0}, c_{1}, \ldots, c_{n}, \ldots
$$

a countably infinite enumeration of atoms not already present in the syntax. Suppose $C$ is a subset of $\left\{c_{i} \mid i \in \omega\right\}$. Suppose $\Gamma, A$ are assertions from the syntax extended by $C$, and that $\mathcal{F}$ is a theory (i.e. a subset of assertions) of the extended syntax. We use

$$
\Gamma \vdash_{\mathcal{F}}^{C} A
$$

to mean the sequent is provable in the proof system for the extended syntax, using the assertions in $\mathcal{F}$ as axioms. A judgment $\Gamma \vdash A$ means a sequent is provable in the proof system of the original assertion language, without extra atoms.

Lemma 25 Let $B$ be a closed assertion and $\mathcal{F}$ a theory in a syntax extended by atoms $C \subseteq\left\{c_{i} \mid i \in \omega\right\}$. Then

$$
\Gamma \vdash_{\mathcal{F} \cup\{B\}}^{C} A \quad \text { iff } \quad \Gamma, B \& \mathbf{1} \vdash_{\mathcal{F}}^{C} A .
$$

Proof " $\Rightarrow$ " By induction on the size of derivations of $\Gamma \vdash_{\mathcal{F} \cup\{B\}}^{C} A$, considering the final rule used.

" $\Leftarrow$ " From $\vdash_{\mathcal{F} \cup\{B\}} B$ and $\vdash \mathbf{1}$ follows $\vdash_{\mathcal{F} \cup\{B\}} B \& \mathbf{1}$ and so the results from hypothesis and cut. 
Definition 26 Let $C \subseteq\left\{c_{i} \mid i \in \omega\right\}$. A subset $\mathcal{F}$ of closed assertions, in the syntax extended by atoms $C$, is called a prime theory iff

(i) $\mathrm{F} \notin \mathcal{F}$,

$A_{1} \oplus A_{2} \in \mathcal{F} \Rightarrow A_{1} \in \mathcal{F}$ or $A_{2} \in \mathcal{F}$,

$\oplus_{x} A \in \mathcal{F} \Rightarrow A[M / x] \in \mathcal{F}$, for some (necessarily closed) marking term $M$.

(ii) $\mathcal{F}$ is deductively closed, i.e. A closed and $\vdash_{\mathcal{F}}^{C} A \Rightarrow A \in \mathcal{F}$.

Lemma 27 (Existence of prime theories). Let $A$ be an assertion and $T$ a subset of closed assertions in the original syntax, for which

$$
\forall_{T} A \text {. }
$$

Then, there is a prime theory $\mathcal{F}$, consisting of assertions over the syntax extended by some $C \subseteq\left\{c_{i} \mid i \in \omega\right\}$, such that

$$
T \subseteq \mathcal{F} \text { and } \nvdash_{\mathcal{F}}^{C} A
$$

Proof As the atoms and variables form countable sets we can enumerate all the assertions

$$
A_{0}, A_{1}, \ldots, A_{n}, \ldots
$$

of the syntax extended by atoms $\left\{c_{i} \mid i \in \omega\right\}$.

By induction, for $n \in \omega$, we define a chain of deductively - closed theories $\mathcal{F}_{n}$ with new atoms $C_{n}$, such that

$$
T \subseteq \mathcal{F}_{n} \text { and } \nvdash_{\mathcal{F}_{n}}^{C_{n}} A
$$

Take $\mathcal{F}_{0}=\left\{B \mid \vdash_{T} B\right\}$. Clearly $T \subseteq \mathcal{F}_{0}$ and, by assumption, $\nvdash_{\mathcal{F}_{0}} A$.

Assuming $\mathcal{F}_{n}$ is deductively closed, includes $T$, and $\nvdash_{\mathcal{F}_{n}}^{C_{n}} A$, define $\mathcal{F}_{n+1}$ according to the following cases:

(i) $n$ is even, and there is an assertion $B_{1} \oplus B_{2} \in \mathcal{F}_{n}$ with $B_{1} \notin \mathcal{F}_{n}, B_{2} \notin$ $\mathcal{F}_{n}$.

(ii) $n$ is odd, and there is an assertion $\oplus_{x} B \in \mathcal{F}_{n}$ with $B[M / x] \notin \mathcal{F}_{n}$ for any closed marking terms $M$ in the syntax over atoms extended by $C_{n}$. 
(iii) neither (i) nor (ii) applies.

In case (iii), define $C_{n+1}=C_{n}$ and $\mathcal{F}_{n+1}=\mathcal{F}_{n}$.

In case (i), take the earliest assertion in the enumeration $B_{1} \oplus B_{2} \in \mathcal{F}_{n}$ and $B_{1} \notin \mathcal{F}_{n}$ and $B_{2} \notin \mathcal{F}_{n}$. As $\mathcal{F}_{n}$ is deductively-closed, $\left(B_{1} \oplus B_{2}\right) \& \mathbf{1} \in \mathcal{F}_{n}$, so

$$
\left(B_{1} \& \mathbf{1}\right) \oplus\left(B_{2} \& \mathbf{1}\right) \in \mathcal{F}_{n}
$$

by the $\&$ - $\oplus$-distributivity law. Suppose

$$
\vdash_{\mathcal{F}_{n}, B_{1}}^{C_{n}} A \text { and } \vdash_{\mathcal{F}_{n}, B_{2}}^{C_{n}} A
$$

Then, by lemma 25 ,

$$
B_{1} \& \mathbf{1} \vdash_{\mathcal{F}_{n}}^{C_{n}} A \text { and } B_{2} \& \mathbf{1} \vdash_{\mathcal{F}_{n}}^{C_{n}} A
$$

Hence $\left(B_{1} \& \mathbf{1}\right) \oplus\left(B_{2} \& \mathbf{1}\right) \vdash_{\mathcal{F}_{n}}^{C_{n}} A$. But this implies $\vdash_{\mathcal{F}_{n}}^{C_{n}} A$, a contradiction. Thus

$$
\nvdash_{\mathcal{F}_{n}, B_{1}}^{C_{n}} A \text { or } \nvdash_{\mathcal{F}_{n}, B_{2}}^{C_{n}} A
$$

Supposing, for instance, $\nvdash_{\mathcal{F}_{n}, B_{1}}^{C_{n}}, A$, take $\mathcal{F}_{n+1}$ to be $\left\{D\right.$ closed $\left.\mid \vdash_{\mathcal{F}_{n}, B_{1}}^{C_{n}}, D\right\}$ and $C_{n+1}=C_{n}$.

In case (ii), take the earliest, according to the enumeration, $\oplus_{x} B \in \mathcal{F}_{n}$ for which $B[M / x] \notin \mathcal{F}_{n}$ for all marking terms $M$, and where $x$ is not a free variable of $A$. As $\mathcal{F}_{n}$ is deductively-closed, $\left(\oplus_{x} B\right) \& \mathbf{1} \in \mathcal{F}_{n}$, so

$$
\bigoplus_{x}(B \& \mathbf{1}) \in \mathcal{F}_{n}
$$

Let $c$ be the first new atom in the list $c_{0}, c_{1}, \ldots$ which is not in $C_{n}$. Define $C_{n+1}=C_{n} \cup\{c\}$ and $\mathcal{F}_{n+1}$ to consist of all closed assertions in the deductive closure of $\mathcal{F}_{n} \cup\{B[c / x]\}$, i.e.

$$
\mathcal{F}_{n+1}=\left\{D \text { closed } \mid \vdash_{\mathcal{F}_{n}, B[c / x]}^{C_{n+1}} D\right\} .
$$

We must check that $\nvdash_{\mathcal{F}_{n+1}}^{C_{n+1}} A$. To this end, assume otherwise, that $\vdash_{\mathcal{F}_{n}, B[c / x]}^{C_{n+1}}$ $A$. Then, by lemma 25 ,

$$
B[c / x] \& \mathbf{1} \vdash_{\mathcal{F}_{n}}^{C_{n+1}} A .
$$

As $c$ does not appear in $C_{n}$ or $\mathcal{F}_{n}$,

$$
B \& \mathbf{1} \vdash_{\mathcal{F}_{n}}^{C_{n}} A
$$


To obtain the proof of this sequent, replace all occurrences of the new atom $c$ in the proof of $B[c / x] \& \mathbf{1} \vdash_{\mathcal{F}_{n}}^{C_{n+1}} A$ by a new variable one which does not appear anywhere in the proof - and finally use (Subst.) to replace this variable by $x$ using the fact that renaming bound variables preserves logical equivalence. But now we can deduce

$$
\bigoplus_{x}(B \& \mathbf{1}) \vdash_{\mathcal{F}_{n}}^{C_{n}} A
$$

But $\oplus_{x}(B \& \mathbf{1}) \in \mathcal{F}_{n}$ and $\mathcal{F}_{n}$ is deductively-closed making $\vdash_{\mathcal{F}_{n}}^{C_{n}} A$, a contradiction. Thus $\nvdash_{\mathcal{F}_{n+1}}^{C_{n+1}} A$, as required.

In this way, we inductively define a chain of theories $\mathcal{F}_{n}$ over the syntax extended by $C_{n}$, such that

$$
C_{n} \subseteq C_{n+1} \text { and } \mathcal{F}_{n} \subseteq \mathcal{F}_{n+1}
$$

with $T \subseteq \mathcal{F}_{0}$. Finally take $C=\cup_{n \in \omega} C_{n}$ and $\mathcal{F}=\cup_{n \in \omega} \mathcal{F}_{n}$ to form the required prime theory.

Assume a prime theory $\mathcal{F}$ with additional atoms $C$. Construct a net $N$ from $\mathcal{F}$ by taking:

- Places to be the original those atoms, including those of $C$.

- Transitions as those pairs $\left(M, M^{\prime}\right)$ of multisets of places for which $\widehat{M} \vdash{ }_{\mathcal{F}}^{C} \widehat{M}^{\prime}$.

We use $\mathcal{M}$ to represent the set of all markings of the net $N$. (Note the markings coincide with the closed marking terms of $\mathcal{F}$.)

Lemma 28 For markings $M, M^{\prime}$ in the atomic net $N$,

$$
M \rightarrow M^{\prime} \text { in } N \quad \text { iff } \quad M \vdash_{\mathcal{F}}^{C} M^{\prime} .
$$

Proof The proof of "iff" is like that for lemma 9.

Regarding atomicity of the net. Suppose $M \rightarrow \underline{0}$, for $M \in \mathcal{M}$. Then $M \vdash_{\mathcal{F}}^{C}$ 1. Certainly

$$
M \oplus(M \& \mathbf{1} \multimap \mathrm{F}) \in \mathcal{F}
$$


and as $\mathcal{F}$ is a prime theory

$$
M \in \mathcal{F} \text { or } M \& \mathbf{1} \multimap \mathrm{F} \in \mathcal{F} .
$$

The case $M \& \mathbf{1} \multimap \mathrm{F} \in \mathcal{F}$ is impossible. To see this assume otherwise, that $M \& \mathbf{1} \multimap \mathrm{F} \in \mathcal{F}$. As $M \vdash_{\mathcal{F}}^{C} \mathbf{1}$, and clearly $M \vdash M$, it follows that $M \vdash_{\mathcal{F}}^{C} M \& \mathbf{1}$ and hence $M \vdash_{\mathcal{F}}^{C}$ F. This ensures $\vdash_{\mathcal{F}}^{C}(M \multimap \mathrm{F})$ which together with the primeness axiom

$$
(M \multimap \mathrm{F}) \vdash \mathrm{F}
$$

yields $\vdash_{\mathcal{F}}^{C}$ F. I.e. $F \in \mathcal{F}$-contradicting $\mathcal{F}$ beeing a prime theory. The case $M \& \mathbf{1} \multimap \mathrm{F} \in \mathcal{F}$ is thus impossible.

$M \in \mathcal{F}$, where $M \vdash_{\mathcal{F}}^{C} \mathbf{1}$. Thus $M \rightarrow \underline{0}$ in the net. The net $N$ is therefore atomic.

We need the following facts:

\section{Lemma 29}

(i) Let $B$ be an assertion with $\mathrm{FV}(B) \subseteq\{x\}$. Let the assertions $\Gamma$ not include $x$ as a free variable. Then

$$
\left(\forall M \in \mathcal{M} . \Gamma \vdash_{\mathcal{F}}^{C} B[M / x]\right) \Rightarrow \Gamma \vdash_{\mathcal{F}}^{C} \underset{x}{\mathbb{\&}} B
$$

(ii) Let $B$ be an assertion with $\mathrm{FV}(B) \subseteq\left\{x_{1}, \ldots, x_{k}\right\}$, and $\Gamma$ be assertions in which $x_{1}, \ldots, x_{k}$ are not free. Then

$$
\left(\forall M_{1}, \ldots, M_{k} \in \mathcal{M} . \Gamma \vdash_{\mathcal{F}}^{C} B\left[M_{1} / x_{1}, \ldots, M_{k} / x_{k}\right]\right) \Rightarrow \Gamma \vdash_{\mathcal{F}}^{C} B .
$$

Proof (i) We first prove the special case

$$
\left(\forall M \in \mathcal{M} . \vdash_{\mathcal{F}}^{C} A[M / x]\right) \Rightarrow \vdash_{\mathcal{F}}^{C} \underset{x}{\&} A,
$$

where $\mathrm{FV}(A) \subseteq\{x\}$, by contraposition. By atomicity,

$$
\vdash\left(\boldsymbol{\&}_{x} A\right) \oplus \bigoplus_{x}(A \& \mathbf{1} \multimap \mathrm{F}),
$$

where both operands of $\oplus$ are closed, so as $\mathcal{F}$ is a prime theory

$$
\vdash_{\mathcal{F}}^{C} \underset{x}{\&} A \text { or } \vdash_{\mathcal{F}}^{C} \bigoplus_{x}(A \& \mathbf{1} \multimap \mathrm{F}) \text {. }
$$


Thus supposing $\nvdash_{\mathcal{F}}^{C} \&_{x} A$ we obtain $\vdash_{\mathcal{F}}^{C} \oplus_{x}(A \& \mathbf{1} \multimap \mathrm{F})$. But as $\mathcal{F}$ is a prime theory, there is then $M \in \mathcal{M}$, such that $\vdash_{\mathcal{F}}^{C}(A[M / x] \& \mathbf{1} \multimap \mathrm{F})$ and thus $\nvdash_{\mathcal{F}}^{C} A[M / x]$.

The more general statement of (i) above follows by taking $A \equiv \otimes \Gamma \multimap B$ where $\Gamma$ does not contain $x$ free and $B$ has at most $x$ free. Note $\Gamma \vdash \&_{x} B$ follows from $\vdash \&_{x}(\otimes \Gamma \multimap B)$.

(ii) follows by indiction using (i) together with the fact that $\&_{x_{k}} B \vdash B$.

Lemma 30 For assertions $\Gamma, B$ and $A$ suppose $\Gamma, B[M / x] \vdash_{\mathcal{F}}^{C} A$ for all $M \in \mathcal{M}$. Assume $\mathrm{FV}(B) \subseteq\{x\}$. Then

$$
\Gamma, \bigoplus_{x} B \vdash_{\mathcal{F}}^{C} A .
$$

Proof We show that if $B[M / x] \vdash_{\mathcal{F}}^{C} A$ for all $M \in \mathcal{M}$ where $\mathrm{FV}(B) \subseteq x$, then $\oplus_{x} B \vdash_{\mathcal{F}}^{C} A$. The seemingly stronger result follows by - -adjunction. Assume $B[M / x] \vdash_{\mathcal{F}}^{C} A$, for all $M \in \mathcal{M}$, and $\mathrm{FV}(B) \subseteq\{x\}$. Let some $M \in \mathcal{M}$ be given. Since $\mathrm{FV}(B[M / x]) \neq \emptyset$ ( $M$ is closed) we can use $(\vdash \&)$ to get $B[M / x] \vdash_{\mathcal{F}}^{C} A^{\prime}$ where $A^{\prime} \equiv \&_{y \in \mathrm{FV}(A)} A$. I.e. $\mathrm{FV}\left(A^{\prime}\right)=\emptyset$ and $\vdash_{\mathcal{F}}^{C} B[M / x] \multimap A^{\prime}$ for all $M \in \mathcal{M}$. By (i) of lemma 29 we then get

$$
\vdash{ }_{\mathcal{F}}^{C} \underset{x}{\boldsymbol{\&}}\left(B \multimap A^{\prime}\right)
$$

and by logic that

$$
\vdash_{\mathcal{F}}^{C}\left(\bigoplus_{x} B\right) \multimap A^{\prime} .
$$

Here we used the logic deduction:

$$
\begin{array}{rlr} 
& \&_{x}\left(B \multimap A^{\prime}\right) \vdash B \multimap A^{\prime} & \text { holds by }(\& \vdash) \text { and (identity), } \\
\Rightarrow & \&_{x}\left(B \multimap A^{\prime}\right), B \vdash A^{\prime} & \\
\Rightarrow & \&_{x}\left(B \multimap A^{\prime}\right), \oplus_{x} B \vdash A^{\prime} & \text { by }(\oplus \vdash) \text { as } x \notin \mathrm{FV}\left(\&_{x}\left(B \multimap A^{\prime}\right), A^{\prime}\right), \\
\Rightarrow & \&_{x}\left(B \multimap A^{\prime}\right) \vdash\left(\oplus_{x} B\right) \multimap A^{\prime} . &
\end{array}
$$

From $\vdash_{\mathcal{F}}^{C}\left(\oplus_{x} B\right) \multimap A^{\prime}$ we get $\oplus_{x} B \vdash_{\mathcal{F}}^{C} A^{\prime}$ and so $\oplus_{x} B \vdash_{\mathcal{F}}^{C} A$ by (\&-adj.).

Now we can relate semantics in the net $N$ to provability in the prime theory $\mathcal{F}$ : 
Lemma 31 For any assertion A, for any marking environment $\rho$,

$$
\llbracket A \rrbracket_{N} \rho=\left\{M \in \mathcal{M} \mid M \vdash_{\mathcal{F}}^{C} A[\rho]\right\} .
$$

Proof By structural induction on $A$.

$A \equiv \mathrm{T}: \llbracket \mathrm{T} \rrbracket_{N} \rho=\mathcal{M}=\left\{M \in \mathcal{M} \mid M \vdash_{\mathcal{F}}^{C} \mathrm{~T}\right\}$.

$A \equiv \mathrm{F}: \llbracket \mathrm{F} \rrbracket_{N} \rho=\emptyset=\left\{M \in \mathcal{M} \mid M \vdash_{\mathcal{F}}^{C} \mathrm{~F}\right\}$ as $\mathrm{F} \notin \mathcal{F}$ and $(M \multimap \mathrm{F}) \vdash \mathrm{F}$.

$A \equiv \mathbf{1}: \llbracket \mathbf{1} \rrbracket_{N} \rho=\{M \in \mathcal{M} \mid M \rightarrow \underline{0}\}=\left\{M \in \mathcal{M} \mid M \vdash_{\mathcal{F}}^{C} \mathbf{1}\right\}$ by lemma 28.

$A \equiv a: \llbracket a \rrbracket_{N} \rho=\{M \in \mathcal{M} \mid M \rightarrow a\}=\left\{M \in \mathcal{M} \mid M \vdash_{\mathcal{F}}^{C} a\right\}$ by lemma 28.

$A \equiv x: \llbracket x \rrbracket_{N} \rho=\{M \in \mathcal{M} \mid M \rightarrow \rho(x)\}=\left\{M \in \mathcal{M} \mid M \vdash_{\mathcal{F}}^{C} \rho(x)\right\}$ by lemma 28 .

$A \equiv A_{1} \otimes A_{2}:$ For $M \in \mathcal{M}$,

$M \in \llbracket A_{1} \otimes A_{2} \rrbracket_{N} \rho$

$\Leftrightarrow \exists M_{1} \in \llbracket A_{1} \rrbracket_{N} \rho, M_{2} \in \llbracket A_{2} \rrbracket_{N} \rho . M \rightarrow M_{1}+M_{2}$,

$\Leftrightarrow \exists M_{1}, M_{2} \in \mathcal{M} . M_{1} \vdash_{\mathcal{F}}^{C} A_{1}[\rho], M_{2} \vdash_{\mathcal{F}}^{C} A_{2}[\rho]$ and $M \vdash_{\mathcal{F}}^{C} M_{1} \otimes M_{2}$,

$\Rightarrow M \vdash_{\mathcal{F}}^{C}\left(A_{1} \otimes A_{2}\right)[\rho]$.

To show " $\Leftarrow$ " as well, we write $A_{1}^{\prime} \equiv A_{1}[\rho]$ and $A_{2}^{\prime} \equiv A_{2}[\rho]$, and use the basic facts that

$$
A_{1}^{\prime} \vdash \oplus_{x_{1}} x_{1} \otimes\left(\left(x_{1} \multimap A_{1}^{\prime}\right) \& \mathbf{1}\right) \text { and } A_{2}^{\prime} \vdash \oplus_{x_{2}} x_{2} \otimes\left(\left(x_{2} \multimap A_{2}^{\prime}\right) \& \mathbf{1}\right) \text {. }
$$

Assuming $M \vdash_{\mathcal{F}}^{C} A_{1}^{\prime} \otimes A_{2}^{\prime}$, we obtain

$$
M \vdash_{\mathcal{F}}^{C}\left(\bigoplus_{x_{1}} x_{1} \otimes\left(\left(x_{1} \multimap A_{1}^{\prime}\right) \& \mathbf{1}\right)\right) \otimes\left(\bigoplus_{x_{2}} x_{2} \otimes\left(\left(x_{2} \multimap A_{2}^{\prime}\right) \& \mathbf{1}\right)\right) .
$$

By distributivity of $\otimes$ over $\oplus$,

$$
M \vdash_{\mathcal{F}}^{C} \bigoplus_{x_{1}} \bigoplus_{x_{2}} x_{1} \otimes\left(\left(x_{1} \multimap A_{1}^{\prime}\right) \& \mathbf{1}\right) \otimes x_{2} \otimes\left(\left(x_{2} \multimap A_{2}^{\prime}\right) \& \mathbf{1}\right) .
$$

By primeness and because $\mathcal{F}$ is a prime theory,

$$
M \vdash_{\mathcal{F}}^{C} M_{1} \otimes\left(\left(M_{1} \multimap A_{1}^{\prime}\right) \& \mathbf{1}\right) \otimes M_{2} \otimes\left(\left(M_{2} \multimap A_{2}^{\prime}\right) \& \mathbf{1}\right)
$$

for some $M_{1}, M_{2} \in \mathcal{M}$.

By atomicity and the fact that $\mathcal{F}$ is a prime theory

$$
\begin{aligned}
& \vdash_{\mathcal{F}}^{C}\left(M_{1} \multimap A_{1}^{\prime}\right) \text { or } \vdash_{\mathcal{F}}^{C}\left(\left(M_{1} \multimap A_{1}^{\prime}\right) \& \mathbf{1} \multimap \mathrm{F}\right) \text { and } \\
& \vdash_{\mathcal{F}}^{C}\left(M_{2} \multimap A_{2}^{\prime}\right) \text { or } \vdash_{\mathcal{F}}^{C}\left(\left(M_{2} \multimap A_{2}^{\prime}\right) \& \mathbf{1} \multimap \mathrm{F}\right) .
\end{aligned}
$$


As $M \nvdash_{\mathcal{F}}^{C} \mathrm{~F}$ and $M_{i} \nvdash_{\mathcal{F}}^{C} \mathrm{~F}$ for $i=1,2$ (by the same argument as in the $A \equiv \mathrm{F}$ case above) we from $(*)$ deduce $\left(M_{i} \multimap A_{i}^{\prime}\right) \& \mathbf{1} \nvdash_{\mathcal{F}}^{C} \mathrm{~F}$ for $i=1,2$, and we must have $\vdash_{\mathcal{F}}^{C}\left(M_{1} \multimap A_{1}^{\prime}\right)$ and $\vdash_{\mathcal{F}}^{C}\left(M_{2} \multimap A_{2}^{\prime}\right)$, i.e. $M_{1} \vdash_{\mathcal{F}}^{C} A_{1}^{\prime}$, and $M_{2} \vdash_{\mathcal{F}}^{C} A_{2}^{\prime}$, as well as $M \vdash_{\mathcal{F}}^{C} M_{1} \otimes M_{2}$. This establishes the required reverse implication, and so equivalence.

$$
\begin{aligned}
A \equiv A_{1} \multimap A_{2}: & M \in \llbracket A_{1} \multimap A_{2} \rrbracket_{N} \rho \\
\Leftrightarrow & \forall M_{1} \in \llbracket A_{1} \rrbracket_{N} \rho . M+M_{1} \in \llbracket A_{2} \rrbracket_{N} \rho . \\
\Leftrightarrow & \forall M_{1} \vdash \mathcal{F} A_{1}[\rho] . M \otimes M_{1} \vdash \mathcal{F} A_{2}[\rho] \text { by induction, } \\
\Leftrightarrow & \forall M_{1} \in \mathcal{M} . M \otimes M_{1} \otimes\left(\left(M_{1} \multimap A_{1}[\rho]\right) \& \mathbf{1}\right) \vdash_{\mathcal{F}}^{C} A_{2}[\rho]
\end{aligned}
$$

where the last equivalence relies on atomicity and the fact that $\mathcal{F}$ is a prime theory. In more detail, writing $A_{1}^{\prime} \equiv A_{1}[\rho], A_{2}^{\prime} \equiv A_{2}[\rho]$, we have

$$
\text { (i) } \vdash_{\mathcal{F}}^{C} M_{1} \multimap A_{1}^{\prime} \quad \text { or } \quad(\text { ii }) \vdash_{\mathcal{F}}^{C}\left(M_{1} \multimap A_{1}^{\prime}\right) \& \mathbf{1} \multimap \mathrm{F}
$$

for any $M_{1} \in \mathcal{M}$. For case (i), $\left(M_{1} \multimap A_{1}^{\prime}\right) \& \mathbf{1} \dashv_{\mathcal{F}}^{C} \mathbf{1}$. In case (ii), $\left(M_{1} \multimap A_{1}^{\prime}\right) \& \mathbf{1} \dashv_{\mathcal{F}}^{C} \mathrm{~F}$.

It follows, by considering the two cases, that for any $M \in \mathcal{M}^{\prime}$

$$
\left(M_{1} \vdash_{\mathcal{F}}^{C} A_{1}^{\prime} \Rightarrow M \otimes M_{1} \vdash_{\mathcal{F}}^{C} A_{2}^{\prime}\right) \text { iff } M \otimes M_{1} \otimes\left(\left(M_{1} \multimap A_{1}^{\prime}\right) \& \mathbf{1}\right) \vdash_{\mathcal{F}}^{C} A_{2}^{\prime} .
$$

Now note that by lemma 30 ,

$$
\forall M_{1} \in \mathcal{M} . M \otimes M_{1} \otimes\left(\left(M_{1} \multimap A_{1}^{\prime}\right) \& \mathbf{1}\right) \vdash_{\mathcal{F}}^{C} A_{2}^{\prime}
$$

$\left.\Leftrightarrow M \otimes \oplus_{x_{1}} x_{1} \otimes\left(\left(x_{1} \multimap A_{1}^{\prime}\right) \& \mathbf{1}\right)\right) \vdash_{\mathcal{F}}^{C} A_{2}^{\prime}$,

$\Leftrightarrow M \otimes A_{1}^{\prime} \vdash_{\mathcal{F}}^{C} A_{1}^{\prime}$ as $A_{1}^{\prime} \nvdash_{\mathcal{F}}^{C} \oplus_{x} x_{1} \otimes\left(\left(x_{1} \multimap A_{1}^{\prime}\right) \& \mathbf{1}\right)$,

$\Leftrightarrow M \vdash_{\mathcal{F}}^{C} A_{1}^{\prime} \multimap A_{2}^{\prime}$ i.e. $M \vdash_{\mathcal{F}}^{C} A_{1}[\rho] \multimap A_{2}[\rho]$, as required.

$$
\begin{aligned}
A \equiv A_{1} \oplus A_{2}: M \in \llbracket A_{1} \oplus A_{2} \rrbracket_{N} \rho & \Leftrightarrow M \in \llbracket A_{1} \rrbracket_{N} \rho \text { or } M \in \llbracket A_{2} \rrbracket_{N} \rho \\
& \Leftrightarrow M \vdash_{\mathcal{F}}^{C} A_{1}[\rho] \text { or } M \vdash_{\mathcal{F}}^{C} A_{2}[\rho] \\
& \Leftrightarrow M \vdash_{\mathcal{F}}^{C} A_{1}[\rho] \oplus A_{2}[\rho] .
\end{aligned}
$$

where primeness and the fact that $\mathcal{F}$ is a prime theory is used in showing " $\Leftarrow$ "; the other direction " $\Rightarrow$ " follows directly from the proof system.

$A \equiv A_{1} \& A_{2}$ :

$$
\begin{aligned}
M \in \llbracket A_{1} \& A_{2} \rrbracket_{N} \rho & \Leftrightarrow M \in \llbracket A_{1} \rrbracket_{N} \rho \text { and } M \in \llbracket A_{2} \rrbracket_{N} \rho \\
& \Leftrightarrow M \vdash_{\mathcal{F}}^{C} A_{1}[\rho] \text { and } M \vdash_{\mathcal{F}}^{C} A_{2}[\rho] \text { by induction, } \\
& \Leftrightarrow M \vdash_{\mathcal{F}}^{C} A_{1}[\rho] \& A_{2}[\rho] .
\end{aligned}
$$




$$
\begin{aligned}
& A \equiv \oplus_{x} B: \\
& M \in \llbracket \oplus_{x} B \rrbracket_{N} \rho \Leftrightarrow \exists M^{\prime} \in \mathcal{M} . M \in \llbracket B \rrbracket_{N} \rho\left[M^{\prime} / x\right] \\
& \Leftrightarrow \exists M^{\prime} \in \mathcal{M} . M \vdash_{\mathcal{F}}^{C} B\left[\rho\left[M^{\prime} / x\right]\right] \text { by induction, } \\
& \Leftrightarrow M \vdash_{\mathcal{F}}^{C}\left(\oplus_{x} B\right)[\rho] .
\end{aligned}
$$

where " $\Rightarrow$ " follows directly from the proof system, whereas " $\Leftarrow$ " relies on primeness and $\mathcal{F}$ being a prime theory.

$$
\begin{aligned}
A \equiv \mathbb{\&}_{x} B: & \\
M \in \llbracket \&_{x} B \rrbracket_{N} \rho & \Leftrightarrow \forall M^{\prime} \in \mathcal{M} . M \in \llbracket B \rrbracket_{N} \rho\left[M^{\prime} / x\right] \\
& \Leftrightarrow \forall M^{\prime} \in \mathcal{M} . M \vdash_{\mathcal{F}}^{C} B\left[\rho\left[M^{\prime} / x\right]\right] \text { by induction, } \\
& \Leftrightarrow M \vdash_{\mathcal{F}}^{C}\left(\&_{x} B\right)[\rho] \text { by (i) of lemma } 29 .
\end{aligned}
$$

\section{Theorem 32 (Completeness).}

Let $A, B$ and $\Gamma \equiv B_{1}, \ldots, B_{n}$ be assertions and $T$ consist of closed assertions in the original syntax. Then,

$$
\Gamma \models_{T} A \quad \text { iff } \quad \Gamma \vdash_{T} A .
$$

Proof As $\multimap$ is present as a constructor on assertions it is clearly sufficient to show

$$
\models_{T} A \text { iff } \vdash_{T} A .
$$

The "if" direction is shown by induction on the proof of $\vdash_{T} A$. To show the "only if" direction we prove its contraposition:

$$
\forall_{T} A \Rightarrow \forall_{T} A \text {. }
$$

Suppose $A$ is an assertion with free variables $x_{1}, \ldots, x_{k}$. Suppose $\nvdash_{T} A$. Then there is a prime theory $\mathcal{F} \supseteq T$ over additional constants $C$ such that $\nvdash_{\mathcal{F}}^{C} A$. Let $N$ be the net constructed from $\mathcal{F}$; let $\mathcal{M}$ be the set consisting of its markings. As we now show, $\nvdash_{N} A$. Suppose otherwise, that $\models_{N} A$. Recall

$$
\llbracket A \rrbracket_{N} \rho=\left\{M \in \mathcal{M} \mid M \vdash_{\mathcal{F}}^{C} A[\rho]\right\}
$$

by lemma 31. Hence as $\models_{N} A$ means $\underline{0} \in \llbracket A \rrbracket_{N} \rho$ for all environments $\rho$, we see

$$
\vdash_{\mathcal{F}}^{C} A[\rho] \text { for all environments } \rho \text {. }
$$

Therefore $\vdash_{\mathcal{F}}^{C} A\left[M_{1} / x_{1}, \ldots, M_{k} / x_{k}\right]$ for all $M_{1}, \ldots, M_{k} \in \mathcal{M}$. Hence by (ii) of lemma $29, \vdash_{\mathcal{F}}^{C} A$, a contradiction. Thus $N$ is an atomic net satisfying all axioms of the theory $T$ and yet $\nvdash_{N} A$. Hence $\nvdash_{T} A$, as required. 


\section{References}

[AV88] Samson Abramsky and Steve Vickers. Linear Process Logic. Notes by Steve Vickers, 1988.

[Bro89] Carolyn Brown. Relating Petri Nets to Formulae of Linear Logic. Technical Report ECS LFCS 89-87, University of Edinburgh, 1989.

[EW90] Uffe Henrik Engberg and Glynn Winskel. Petri Nets as Models of Linear Logic. In CAAP '90, Coll. on Trees in Algebra and Programming Copenhagen, Denmark, May 15-18, pages 147161. Springer-Verlag (LNCS 431), 1990.

[GG89] Carl Gunter and Vijay Gehlot. Nets as Tensor Theories. Technical Report MS-CIS-89-68, University of Pennsylvania, October 1989 .

[Gir87] Jean-Yves Girard. Linear Logic. Theoretical Computer Science, 50(1):1-102, 1987.

[GL87] Jean-Yves Girard and Yves Lafont. Linear Logic and Lazy Computation. In Proc. TAPSOFT 87 (Pisa), vol. 2, pages 52-66. Springer-Verlag (LNCS 250), 1987.

[Laf88] Yves Lafont. The Linear Abstract Machine. Theoretical Computer Science, 59:157-180, 1988.

[MOM91] Narciso Martí-Oliet and José Meseguer. From Petri Nets to Linear Logic: a Survey. International Journal of Foundations of Computer Science, 2(4):297-399, 1991.

[Rei85] Wolfgang Reisig. Petri Nets, An Introduction, volume 4 of EATCS Monographs on Theoretical Computer Science. Springer-Verlag, 1985.

[Ros90] Kimmo I. Rosenthal. A Note on Girard Quantales. Chaiers de Top. et Géom. Diff. Cat., 31(1):3-11, 1990.

[Sam] G. Sambin. . Manuscript reported to us by Per Martin-Löf.

[Yet90] David N. Yetter. Quantales and (Non-Commutative) Linear Logic. The Journal of Symbolic Logic, 55:41-64, 1990. 